\title{
Evaluation of the individual workload perception of the personnel in food service system
}

\author{
Hulya Kamarli Altun and Nilgun Seremet Kurklu \\ Akdeniz University, Faculty of Health Sciences, Department of Nutrition and Dietetics, Antalya, Turkey
}

\begin{abstract}
Introduction: Organizational factors, insufficient wages, inadequate work performance of their co-workers and lack of physical working environment in the hospital food service system (FSS) cause the increase of the workload. This study aims to determine the individual workload perception of the FSS personnel and to ascertain whether the individual workload perception differs across demographic variables and occupational groups.
\end{abstract}

Materials and Methods: The population of this descriptive study consists of the personnel working in the kitchen of the Akdeniz University Hospital, and the sample consists of 87 people who accepted to participate in the study by filling out the questionnaire forms on a voluntary basis. Individual Workload Perception Scale was used to evaluate the personnel's perception on their work environment and Personal Information Form was used to detect the participants' data related to the independent variables.

Results: Approximately half of the individuals (47.1\%) participating in the study were aged 31-40 years (33.5 \pm 7.32$)$. 56.3\% of the individuals were waiter and three quarters of the individuals worked in shifts. Total individual workload perception of the FSS personnel was detected as $64.7 \pm 15.25$ on average. The mean score of the colleague support sub-group of the individuals was the highest and the mean score of the unit support was the lowest. It was determined that FSS personnel did not display a statistically significant difference across age groups, gender, marital status, educational status and individual workload sub-groups. It was only seen that there was a significant association between the work distribution of the individuals and the colleague\&unit support ( $\mathrm{p}<0.05$ ). A positively significant correlation was detected in FSS personnel between colleague support $(\mathrm{r}=0.859)$, unit support $(\mathrm{r}=0.473)$, work environment perception $(r=0.647)$ and intention to resume current work $(r=0.434)$ subscales and total individual workload perception scale $(\mathrm{p}<0.01)$.

Discussion: The workload of the FSS personnel working in the hospital was found to be high, and it is necessary to evaluate the individual workload and develop solutions for the provision of dietary services in a safe, high quality and efficient manner.

\section{Conflict of Interest}

There is no conflict of interest. 